\title{
Conference Report for the Advances in Architectural Geometry (AAG) 2014
}

\author{
Niloy J. Mitra
}

Published online: 8 January 2015

(C) Kim Williams Books, Turin 2015

\begin{abstract}
Advances in Architectural Geometry (AAG) is a conference where both theoretical and practical work linked to new geometrical developments is presented. The conference aims to gather the diverse components of the contemporary architectural tendencies that push the building envelope towards free form and respond to the multiple current design challenges. It involves architects, engineers, mathematicians, computer scientists, and contractors. This conference report summarizes the recently concluded AAG 2014 held in London.
\end{abstract}

Keywords Architectural geometry - Computational design · Algorithms · Design and practice

The fourth edition of the Advances in Architectural Geometry (AAG) 2014 was recently held at the University College London from 18 to 21 September. Attended by over 300 participants both from the industry and academia, AAG14 featured a mix of architects, computer scientists, engineers, and mathematicians. Started in 2008, AAG is now a leading venue for dissemination of knowledge and experience relating to architectural geometry across the communities of architecture, design, structural engineering, computer science, and applied geometry.

\footnotetext{
N. J. Mitra $(\bowtie)$

Department of Computer Science, University College London, Gower Street, London WC1E 6BT, UK

e-mail: n.mitra@cs.ucl.ac.uk
} 




Prof. Mark Pauly, EPFL during his keynote lecture at AAG2014

The focus of AAG14 was on developments in computational design in the context of architectural geometry, with leading practitioners presenting their thoughts on current design challenges, new computational methodologies, and trends in the context of cutting edge architectural practice. The conference comprised of 2 days of technical paper presentations, followed by another 2 days of hands-on workshops.

As the main focus, 24 peer-reviewed papers were presented ranging in topics from computational design, 3D fabrication techniques, computational construction sequences, structural analysis, etc. The best papers were awarded to: "Surface panelization using periodic conformal maps" by ThiloRörig, Stefan Sechelmann, AgataKycia and Moritz Fleischmann for the best technical paper, and to the "Interlocking Folded Plate-Integrated Mechanical Attachment for Structural Timber Panels" by Christopher Robeller, Andrea Stitic, Paul Mayencourt and Yves Weinand for the best design practice and fabrication paper. The technical paper chairs were Jan Knippers, Philippe Block, Wenping Wang who were responsible for selecting the accepted papers with the help of the International Program. In addition, 24 posters were accepted, which were presented in a poster session where the authors and the participants engaged in extensive discussion and feedback.

AAG14 featured four high-profile keynote speakers, who all gave inspiring talks and provided glimpses to exciting opportunities lying ahead: Cristiano Ceccato, who is well-known for his contribution in many iconographic buildings of Zaha Hadid Architects, described the development of rule-based design processes and parametric form finding in digital design processes. Mark Pauly, Professor of Computer Science at the Ecole Polytechnique Federal de Lausanne, brought us back to core studies in geometry and computational approaches to form-finding. Daniel Bossia, Director at AKT II and head of the specialist team P.art ${ }^{\circledR}$ and an expert in computational and structural design with extensive knowledge in programming, shared his many experiences and opinions on form finding and non-linear analysis 
and illustrated his views using the evolution of many legendary design projects as case studies. Finally, Behrokh Khoshnev is, Professor at the University of Southern California, who is widely regarded as a pioneer in contour printing, described his vision to automate the construction of single buildings or colonies of buildings in the very near future.

The conference also featured 10 workshops, spread across 2 days that were extremely well attended with more than a total of 130 participants. The workshops spanned a range of topics including form-finding of twisted interlaced structures, tessellation, computational design and panelization, first person holograms, etc. The workshop program was selected and coordinated by the workshop chair Alexander Schiftner.

Further details about the conference, along with the list of accepted papers, talk abstracts, workshop descriptions, list of participant, etc. can be found at the conference webpage: http://www.architecturalgeometry.org/aag14/.

After four intensive and exciting days, AAG14 was concluded with the participants already planning to return back for the next edition of AAG scheduled for 2016 at Zurich.

Niloy J. Mitra is a Professor of Geometry Processing in the Department of Computer Science at University College London (UCL). He received his $\mathrm{PhD}$ degree and Masters in Electrical Engineering from the Stanford University. His research interests include shape understanding, structure-aware geometry processing, architectural geometry, fabrication-aware design, and geometric modeling in general. His works have twice been selected as Research Highlights in Communications of ACM and has received best paper awards at Symposium on Geometry Processing (SGP) and EURO GRAPHICS. He received the ACM SIGGRAPH Significant New Researcher Award in 2013 for his work on integrating form and function in 3D geometry. 